\title{
Molecular Epidemiology and Dynamics of Pseudomonas aeruginosa Populations in Lungs of Cystic Fibrosis Patients $\nabla$
}

\author{
Lars Jelsbak, ${ }^{1}$ Helle Krogh Johansen, ${ }^{2,3}$ Anne-Louise Frost, ${ }^{1}$ Regitze Thøgersen, ${ }^{1}$ Line E. Thomsen, ${ }^{4}$ \\ Oana Ciofu, ${ }^{3}$ Lei Yang, ${ }^{1}$ Janus A. J. Haagensen, ${ }^{1}$ Niels Høiby, ${ }^{2,3}$ and Søren Molin ${ }^{1 *}$ \\ Infection Microbiology Group, BioCentrum-DTU, Technical University of Denmark, Lyngby, Denmark ${ }^{1}$; Copenhagen CF Center \\ Department of Clinical Microbiology, Rigshospitalet, ${ }^{2}$ and Institute of Medical Microbiology and Immunology, Panum Institute, \\ University of Copenhagen, ${ }^{3}$ Copenhagen, Denmark; and Department of Veterinary Pathobiology, The Royal Veterinary and
} Agricultural University, DK-1870 Frederiksberg C, Denmark ${ }^{4}$

Received 10 August 2006/Returned for modification 31 October 2006/Accepted 16 January 2007

\begin{abstract}
The ability to establish lifelong persistent infections is a fundamental aspect of the interactions between many pathogenic microorganisms and their mammalian hosts. One example is chronic lung infections by the opportunistic pathogen Pseudomonas aeruginosa in cystic fibrosis (CF) patients. This infection process is associated with extensive genetic adaptation and microevolution of the infecting bacteria. Through investigations of $P$. aeruginosa populations and infection dynamics in a group of CF patients followed at the Danish CF Clinic in Copenhagen, we have identified two distinct and dominant clones that have evolved into highly successful colonizers of CF patient airways. A significant component of the evolutionary success of these two clones has been their efficient transmissibility among the CF patients. The two clones have been present and transmitted among different $\mathrm{CF}$ patients for more than 2 decades. Our data also suggest that the $\boldsymbol{P}$. aeruginosa population structure in the CF patient airways has been influenced by competition between different clones and that the two dominant clones have been particularly competitive within the lungs, which may add to their overall establishment success. In contrast, we show that adaptive traits commonly associated with establishment of chronic $P$. aeruginosa infections of CF patients, such as transition to the mucoid phenotype and production of virulence factors, play minor roles in the ability of the two dominant clones to spread among patients and cause long-term chronic infections. These findings suggest that hitherto-unrecognized evolutionary pathways may be involved in the development of successful and persistent $P$. aeruginosa colonizers of CF patient lungs.
\end{abstract}

Pseudomonas aeruginosa, the most common pathogen associated with morbidity and mortality in patients suffering from cystic fibrosis (CF), causes chronic lung infections that, once established, are difficult to eradicate even with intensive antibiotic treatment (15).

The development of chronic lung infections follows a characteristic pattern. Typically, a period of intermittent colonization with $P$. aeruginosa early in the life of the patient precedes the establishment of chronic infections $(5,17)$. These earlyinfecting strains typically resemble those found in the environment, being nonmucoid, fast growing, and relatively susceptible to antibiotics (16). This stage is temporary, and eventually the airways of nearly all patients become permanently colonized by $P$. aeruginosa.

During chronic infection, the bacterial population adapts to the environment in the CF patient airways. This process correlates with considerable genetic adaptation and accumulation of loss-of-function mutations in specific $P$. aeruginosa genes (40). One common mutation is in the mucA gene, which causes a transition from a nonmucoid to a mucoid, alginate-overproducing phenotype (24). Mucoid $P$. aeruginosa is of special in-

\footnotetext{
* Corresponding author. Mailing address: Infection Microbiology Group, BioCentrum-DTU, Technical University of Denmark, Building 301, 2800 Lyngby, Denmark. Phone: 454525 2513. Fax: 4545887328. E-mail: sm@biocentrum.dtu.dk.

${ }^{\nabla}$ Published ahead of print on 29 January 2007.
}

terest as an indicator of chronic infection and, because of the association with poor prognosis, deteriorating lung function and tissue damage (33). Early intervention and aggressive antimicrobial chemotherapy after initial detection of $P$. aeruginosa have been shown to delay the transition from intermittent to chronic infection in the majority of patients $(11,27,42)$.

The prevailing view of the infection process is that most of the infections are clonal and that individual $\mathrm{CF}$ patients acquire unique $P$. aeruginosa strains independently, presumably from diverse environmental sources $(16,22,35,36)$. However, several recent reports have shown the occurrence of aggressive and transmissible strains of $P$. aeruginosa in patients attending CF centers in Europe and Australia (2, 3, 7, 8, 10, 19, 25, 29-31, 34, 39), and some transmissible clones may in fact be more virulent than the usual infecting types, potentially resulting in poor prognoses for the patients $(1,3,28,37)$.

We have investigated these different aspects of $P$. aeruginosa infections in a defined group of CF patients from the CF Center in Copenhagen, Denmark. In particular, we have used molecular epidemiological tools to better understand how the bacteria infect and establish persistent infections in CF patient airways, from the early intermittent colonization in children and young adults to the chronic state of infection in older patients.

\section{MATERIALS AND METHODS}

Bacterial isolates. The $P$. aeruginosa strain collection examined consisted of the following: 122 isolates from $7 \mathrm{CF}$ patients with long-term chronic infection 
TABLE 1. Characteristics of patients included in this study

\begin{tabular}{|c|c|c|c|c|c|c|}
\hline Patient type & Patient no. & Yr of birth & Gender & $\begin{array}{c}\text { Yr chronic } \\
\text { infection began } \\
\text { (duration [yr]) }\end{array}$ & $\begin{array}{c}\text { Median } \\
\text { P. aeruginosa-specific } \\
\text { precipitins (range) } \\
\text { last } 5 \mathrm{yr}\end{array}$ & Bacteriology ${ }^{a}$ \\
\hline \multirow[t]{13}{*}{ Intermittently colonized } & B1 & 1990 & M & $-^{c}$ & $1(0-1)$ & NM \\
\hline & B4 & 2004 & M & - & $0(0-0)$ & NM \\
\hline & B6 & 2002 & $\mathrm{~F}$ & - & $1(0-1)$ & NM \\
\hline & B7 & 1995 & M & - & $0(0-1)$ & NM \\
\hline & B9 & 2001 & $\mathrm{~F}$ & - & $0(0-1)$ & NM \\
\hline & B10 & 1999 & $\mathrm{~F}$ & - & $1(0-2)$ & NM \\
\hline & B12 & 1998 & M & - & $0(0-1)$ & NM \\
\hline & B14 & 1982 & M & - & $0(0-1)$ & NM \\
\hline & B18 & 1988 & $\mathrm{~F}$ & - & $0(0-0)$ & NM \\
\hline & B22 & 1996 & $\mathrm{~F}$ & - & $0(0-0)$ & NM \\
\hline & B23 & 1998 & $\mathrm{~F}$ & - & $0(0-1)$ & M \\
\hline & B29 & 1999 & $\mathrm{~F}$ & - & $0(0-0)$ & NM \\
\hline & B36 & 1993 & $\mathrm{~F}$ & - & $1(0-2)$ & NM \\
\hline \multirow[t]{2}{*}{ Recently chronically infected } & B3 & 2002 & $\mathrm{~F}$ & $2005(<1)$ & $5(0-16)$ & $\mathrm{M} / \mathrm{NM}$ \\
\hline & B19 & 1979 & $\mathrm{~F}$ & $2005(<1)$ & $1(0-23)$ & NM \\
\hline \multirow[t]{7}{*}{ Long-term chronically infected } & p2 & 1966 & $\mathrm{~F}$ & $1976(29)$ & $41(32-45)$ & $\mathrm{M} / \mathrm{NM}$ \\
\hline & p6 & 1975 & $\mathrm{~F}$ & $1984(21)$ & $27(22-29)$ & $\mathrm{NM}^{b}$ \\
\hline & p7 & 1986 & $\mathrm{~F}$ & $1990(15)$ & $17(12-20)$ & $\mathrm{NM}^{b}$ \\
\hline & p8 & 1963 & M & $1976(29)$ & $28(21-30)$ & $\mathrm{M} / \mathrm{NM}$ \\
\hline & p10 & 1963 & M & $1980(25)$ & $25(20-30)$ & $\mathrm{NM}^{b}$ \\
\hline & p11 & 1983 & M & 1993 (12) & $18(13-22)$ & $\mathrm{M} / \mathrm{NM}$ \\
\hline & p16 & 1973 & $\mathrm{~F}$ & $1976(29)$ & $37(35-44)$ & $\mathrm{M} / \mathrm{NM}$ \\
\hline
\end{tabular}

\footnotetext{
${ }^{a}$ M, mucoid; NM, nonmucoid; M/NM, mucoid and nonmucoid.

${ }^{b}$ The bacteriology records showed that mucoid variants have been found (but not stored) in occasional samples in the history of the patients.

$c$ - no chronic infection.
}

and 28 isolates from $15 \mathrm{CF}$ patients who were intermittently colonized or recently chronically infected and who were attending the Danish CF Center, Rigshospitalet, Copenhagen; 11 isolates from non-CF patients obtained from six different departments at Rigshospitalet, Copenhagen; 1 environmental isolate from a sanitary facility in Copenhagen; and 1 reference strain (PA01).

Isolation and identification of $P$. aeruginosa from sputum were done as previously described (15). The sputum samples were obtained by expectoration or endolaryngeal suction, followed by Gram staining and microscopic examination to confirm their origin from the lower airways by the presence of mucus and leukocytes (15). P. aeruginosa was identified by conventional biochemical tests. $P$. aeruginosa isolates from samples obtained during 2005 were isolated on Pseudomonas isolation agar (Difco) containing ampicillin $(100 \mu \mathrm{g} / \mathrm{ml})$. At least one colony of each morphotype present in a sputum sample was analyzed. For samples taken in 2005, we furthermore routinely performed phenotypic screening of 96 colonies per sample for differences in antibiotic resistance profiles and/or colony appearance. Colonies with different phenotypes were chosen for further analysis. All $P$. aeruginosa isolates collected were frozen at $-80^{\circ} \mathrm{C}$.

CF patients. Patient data for the $22 \mathrm{CF}$ patients included in this study are shown in Table 1. As part of the general management structure at the CF Center, all patients were monitored on a monthly basis by evaluation of their clinical status, pulmonary function, and microbiology of lower-airway secretions. Chronic $P$. aeruginosa infection was defined as the persistent presence of $P$. aeruginosa in sputum for 6 consecutive months or less when persistence was combined with the presence of two or more precipitating antibodies against $P$. aeruginosa (18). The number of precipitating antibodies against $P$. aeruginosa in serum from all included patients was determined by crossed immunoelectrophoresis as previously described (14).

$P$. aeruginosa genotyping. Identification of $P$. aeruginosa genotypes was done by single-nucleotide polymorphism (SNP) typing using AT biochips (Clondiag Chip Technologies, Germany). The biochip allows simultaneous detection of 13 SNPs previously identified in highly conserved genomic regions such as oriC, citS, ampC, oprI, fliC, oprL, and alkB2 (26). The theoretical accuracy of using these SNPs for genotyping is $99.7 \%$ (26) (Clondiag Chip Technologies, Germany). In addition to SNP typing, the AT chip allows detection of several gene islands, pathogenicity factors, and variable genes, providing some insight into the repertoire of the accessory genome of a given isolate. We consider isolates with the same SNP pattern to be clones and isolates with the same SNP pattern and the same pattern of accessory genes to be identical strains.

The AT chip assay is based on detection of hybridization of labeled genomic target DNA to a DNA microarray containing a set of 77 specific oligonucleotide probes. The AT chip assay was performed as described by the manufacturer with minor modifications. Briefly, target DNA from P. aeruginosa isolates to be genotyped was amplified and biotin labeled for the AT chip assay by multiplex PCR in 40 cycles of $96^{\circ} \mathrm{C}$ for $60 \mathrm{~s}, 60^{\circ} \mathrm{C}$ for $20 \mathrm{~s}$, and $72^{\circ} \mathrm{C}$ for $40 \mathrm{~s}$ using at set of 77 optimized primer pairs from the manufacturer and Taq polymerase (Sigma). Hybridization of the biotinylated PCR products to the AT chip was done at $60^{\circ} \mathrm{C}$ for $45 \mathrm{~min}$. Subsequent washing and blocking of the AT chip were performed as recommended by the manufacturer. Hybridizing spots were visualized using $3,3^{\prime}, 5,5^{\prime}$-tetramethylbenzidine as the substrate for streptavidin-conjugated horseradish peroxidase. Hybridizing signals were detected using a Zeiss Axioplan-2 microscope equipped with a Coolsnap Pro charge-coupled device camera controlled by Image Pro image analysis software (Media Cybernetics, Silver Spring, MD). Analysis of signal intensities permitted distinction between different SNP variants as well as the absence or presence of variable genes and gene islands. The AT chip system was introduced to us by L. Wiehlmann and B. Tümmler (Medical School, Hannover, Germany).

The clonal relatedness of selected $P$. aeruginosa isolates was also assessed by pulsed-field gel electrophoresis (PFGE) as described previously (20).

Sequencing of $\boldsymbol{m u c A}$ and $\boldsymbol{a l g} \boldsymbol{T}$ genes. A 687-bp fragment covering the entire $m u c A$ region and an 831-bp fragment covering the entire $\operatorname{alg} T$ region were amplified by standard PCR. Purified PCR products were sequenced by Macrogen's (South Korea) sequencing service. Primers used for PCR amplification and sequencing of $m u c A$ were mucA1 (5'-CTCTGCAGCCTTTGTTGCGAGAAG$\left.3^{\prime}\right)$, mucA1rev (5'-CTGCCAAGCAAAAGCAACAGGGAGG-3'), mucA2 (5' GTGCGTCTGTACAACCAGAACGACG-3'), and mucA2rev (5'-GTCGTTC TGGTTGTACAGACGCACG-3'). Primers used for PCR amplification and sequencing of $\operatorname{alg} T$ were algTsek1 (5'-CCTGAGCCCGATGCAATCCATTTTC G-3'), algTsek2 (5'-GGTATGCCTTGATGAAGGCTTCCTGC-3'), algTsek3 (5'-GCTGTATCGGATCGCCATCAACACC-3'), and algTsek4 (5'-GGACAG AGTTTCCTGCAGGGCTTCAC-3').

Caenorhabditis elegans killing assay. The killing of the C. elegans pha1(e2123ts) strain by $P$. aeruginosa isolates was measured on NG agar (slow-killing 


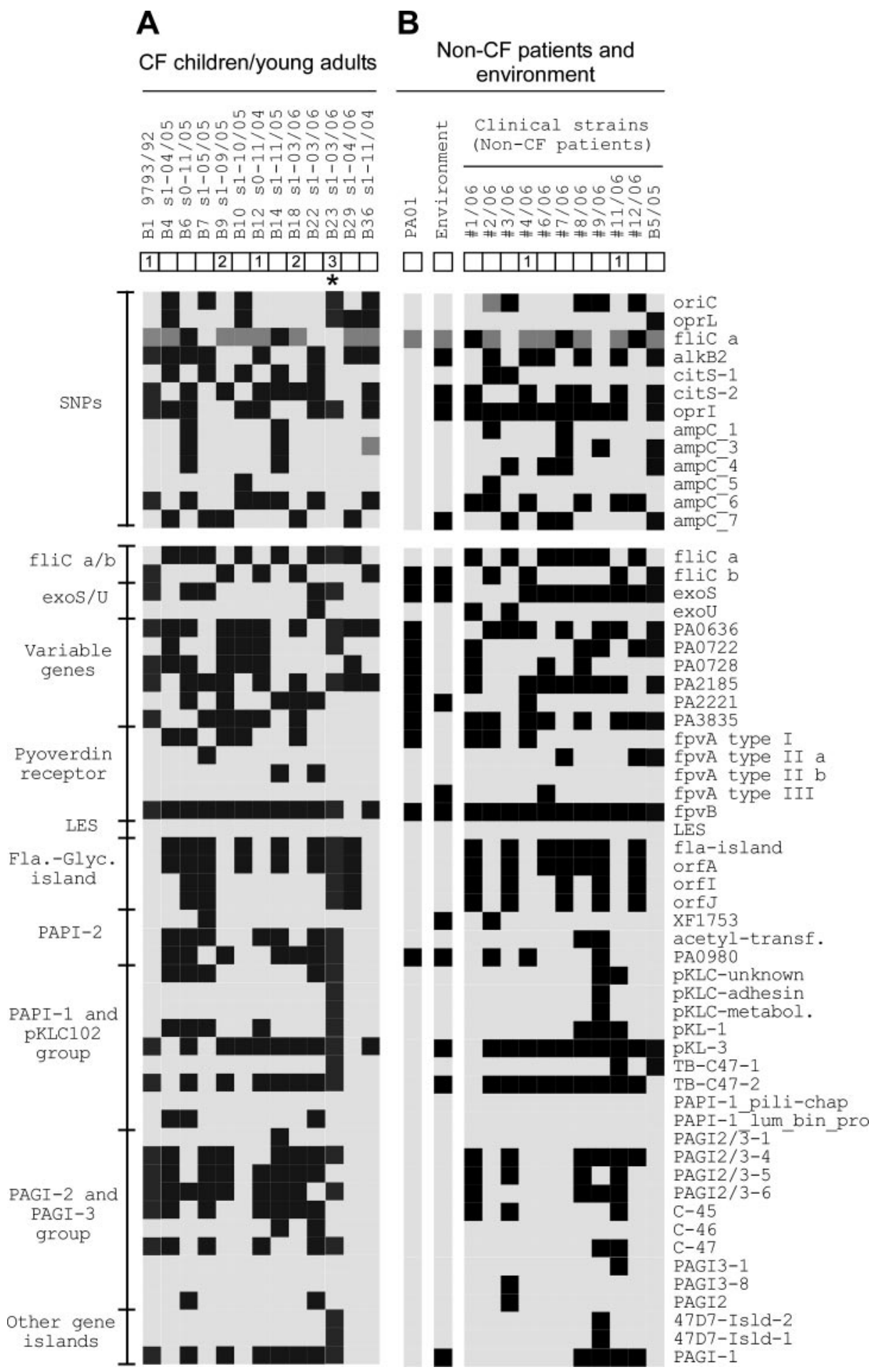

FIG. 1. Genotyping of $P$. aeruginosa isolates. (A) Isolates from young CF patients with intermittent colonization. (B) Clinical isolates from non-CF patients. Boxes below the names of the isolates indicate specific genotype classes. White boxes specify unique genotypes found only once in the data set. Labeled boxes specify that a genotype was found in another patient. The asterisk indicates mucoid isolates. Genotyping was based on SNP typing. In the SNP panel, light gray and black boxes indicate similar and different SNP sequences, respectively, compared to the PA01 sequence. Dark gray boxes indicate SNPs that were not measured. Below the SNP panel, the presence or absence of particular genes or regions in different isolates is shown by black and light gray boxes, respectively. 
assay) essentially as previously described (43). The temperature-sensitive sterile C. elegans pha-1(e2123ts) strain used here grows normally at $15^{\circ} \mathrm{C}$, but the allele is embryonic lethal at $25^{\circ} \mathrm{C}(38)$. Mutation in the pha-1 gene does not affect the immune function of C. elegans (44). The Escherichia coli and C. elegans strains used in this work were provided by the Caenorhabditis Genetics Center (University of Minnesota, Minneapolis).

\section{RESULTS}

High $P$. aeruginosa genotype diversity in intermittently colonized children and young adults with CF. In order to understand the $P$. aeruginosa population dynamics during the early stages of CF lung infection, we first determined the genotypes of a set of $P$. aeruginosa strains isolated from 13 Danish CF patients with intermittent $P$. aeruginosa colonization and with a mean age of 10.3 years (Table 1 ). None of the patients had elevated levels of precipitating antibodies against $P$. aeruginosa, and 12 of the $P$. aeruginosa isolates were nonmucoid and susceptible to standard antipseudomonas antibiotics (data not shown). Mucoid variants were found only in patient B23. Genotyping of the infecting isolates was performed by analyzing SNPs of conserved genomic regions using Clondiag AT biochips, which provide strain discrimination up to a specificity of $99.7 \%$ (Materials and Methods).

Our data show that 8 of the 13 young CF patients were colonized with unique clones, whereas identical $P$. aeruginosa genotypes were isolated from patients $\mathrm{B} 1$ and $\mathrm{B} 12$ and from $\mathrm{B} 9$ and B18 (Fig. 1A). In addition, the same $P$. aeruginosa genotype was isolated from patient B23 and the recently chronically infected patient B3 (cf. Fig. 1A and 3A). We also genotyped a collection of 11 clinical $P$. aeruginosa isolates from non-CF patients admitted to the hospital as well as the reference strain PA01 and an environmental isolate. Again, 11 of these 13 strains represented unique genotypes, whereas the same clone was found in two different non-CF patients; interestingly, the clone was identical to the one found in $\mathrm{CF}$ patients $\mathrm{B} 1$ and $\mathrm{B} 12$ (Fig. 1A and B). Thus, despite some examples of possible cross-infections, our data show that intermittently colonized CF patients have an overall high level of $P$. aeruginosa genotype diversity equivalent to the diversity found in settings other than the CF patient airways. In agreement with previous findings, our data thus show that there is not a specific genotype of $P$. aeruginosa responsible for colonization of young $\mathrm{CF}$ patients $(5,27)$.

Recolonization by $\boldsymbol{P}$. aeruginosa can be caused by identical or different clones. Our genotyping data suggest that most intermittently colonized CF patients acquire unique clones from the environment. Early aggressive antibiotic chemotherapy temporarily eradicates these bacteria; eradication is followed by new colonization events $(11,27,42)$. To further understand the dynamics of these reemerging $P$. aeruginosa colonizations, we genotyped strains isolated from patients in which $P$. aeruginosa was again isolated after a period of no detectable $P$. aeruginosa in sputum samples. From patients B7 and B12 the same $P$. aeruginosa genotypes as those originally isolated and stored as first samples in the hospital collection were identified in subsequent samples (Fig. 2). From patient B6, however, we isolated more than one genotype (Fig. 2). Taken together, these data illustrate that recolonization can be caused either by new strains from the environment or by the same clone, which may have remained transiently undetectable
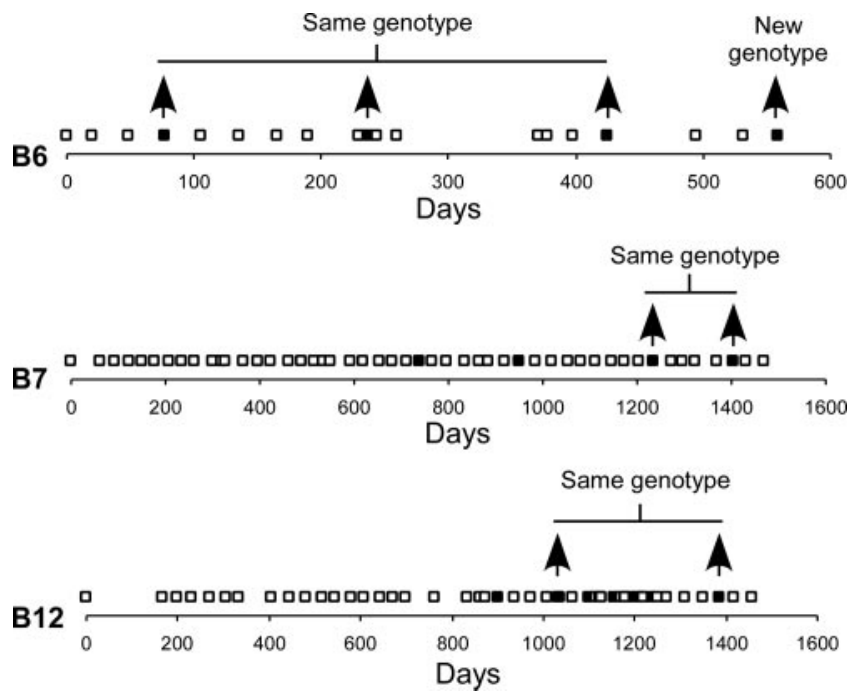

FIG. 2. Time course of $P$. aeruginosa isolation from three intermittently colonized patients. Black and white boxes above the timeline indicate positive and negative cultivation of $P$. aeruginosa from patient samples, respectively.

by culture of sputum or have appeared by reinfection from a specific host or from an environmental source in close association with the patient.

High $\boldsymbol{P}$. aeruginosa genotype diversity among recently diagnosed chronically infected patients. During the course of our studies of intermittent colonizations, two young CF patients (B3 and B19) were excluded from this group as they became chronically infected with $P$. aeruginosa and exhibited an elevated level of precipitins (Table 1). However, this gave us the opportunity to study the initial phase of chronicity. Genotyping of eight sequential isolates from patient B3 over a period of 24 months demonstrated a clonal colonization of initially nonmucoid $P$. aeruginosa followed by the appearance of mucoid variants of the same genotype (Fig. 3A). This genotype is identical to that found in a newly colonized child (B23). From B19 we have so far obtained only one isolate (unique genotype).

Low $P$. aeruginosa genotype diversity among CF patients with long-term chronic infection. We next determined the genotypes of a set of 45 P. aeruginosa strains isolated during 2005 from seven Danish CF patients with long-term chronic $P$. aeruginosa infections. The group of patients studied here has been diagnosed as being chronically infected with $P$. aeruginosa for more than 12 years and are cohort isolated from other patients at the CF Center (Table 1). Our genotype data based on SNP analysis show that the investigated patients were colonized mainly by one clone or very few coexisting clones (Fig. 3B). Interestingly, only five different $P$. aeruginosa genotypes were found among the 45 isolates tested, and considerable overlap in clones among the patients was observed (Fig. 3B). Two genotypes (marked " $r$ " and "b" in Fig. $3 \mathrm{~B}$ ) were particularly dominant and were found in four and two patients, respectively, as well as coexisting in one patient. This finding of relatedness was confirmed by PFGE analysis of selected strains. As shown in Fig. 4, the PFGE data correlate well with the AT biochip results from representative isolates. In a reverse experiment, we used the PFGE pattern for the $b$ geno- 


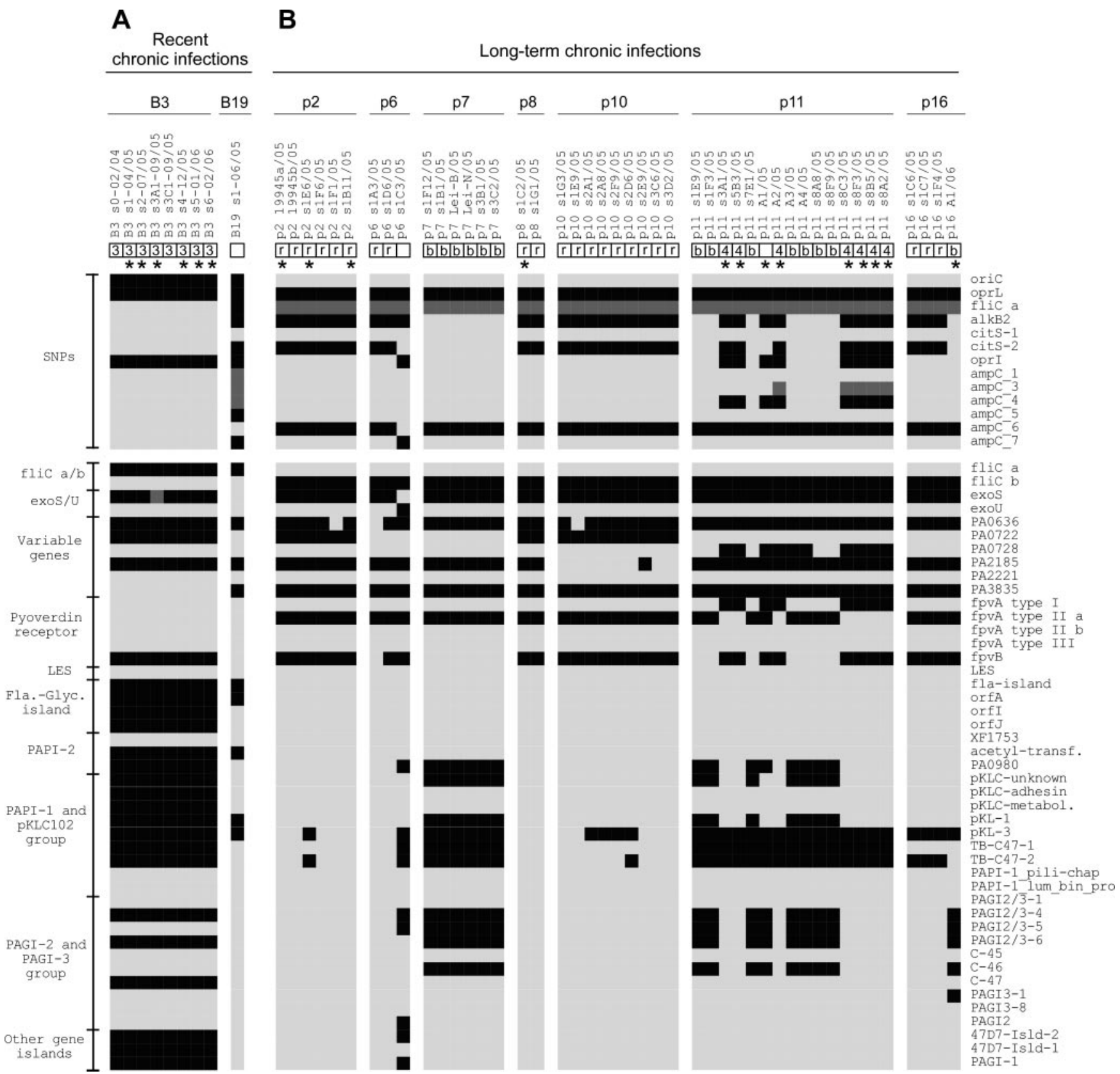

FIG. 3. Genotyping of $P$. aeruginosa isolates from chronic infections. (A) Isolates from young CF patients with recent chronic infection. (B) Isolates from patients with long-term chronic infection. Boxes below the names of the isolates indicate specific genotype classes. White boxes specify unique genotypes found only once in the data set. Labeled boxes specify that a genotype was found in another patient. Asterisks indicate mucoid isolates. Genotyping was based on SNP typing. In the SNP panel, light gray and black boxes indicate similar and different SNP sequences, respectively, compared to the PA01 sequence. Dark gray boxes indicate SNPs that were not measured. Below the SNP panel, the presence or absence of particular genes or regions in different isolates is shown by black and light gray boxes, respectively.

type (Fig. 4) to search our PFGE database. We chose nine isolates from eight different patients with a PFGE pattern that suggested they were of the b genotype. This was in all cases confirmed by SNP typing (data not shown).

These data show that, in contrast to our findings for $\mathrm{CF}$ patients with intermittent colonization or recent chronic infections, an overall low level of $P$. aeruginosa genotype diversity and the existence of two dominant clones characterize the Copenhagen $\mathrm{CF}$ patients with long-term chronic infections.
Genetic variations among clonal isolates. To further characterize the two dominant clones, we examined the presence and absence of variable genomic regions monitored on the AT biochips (Fig. 3B). This revealed some differences, both within isolates from a particular patient and between clones isolated from different patients. For example, among 22 isolates of the $r$ genotype from five of the patients we found differences in 6 of the 45 investigated variable regions on the AT biochip, on the basis of which the 22 clones could be divided into 10 groups 


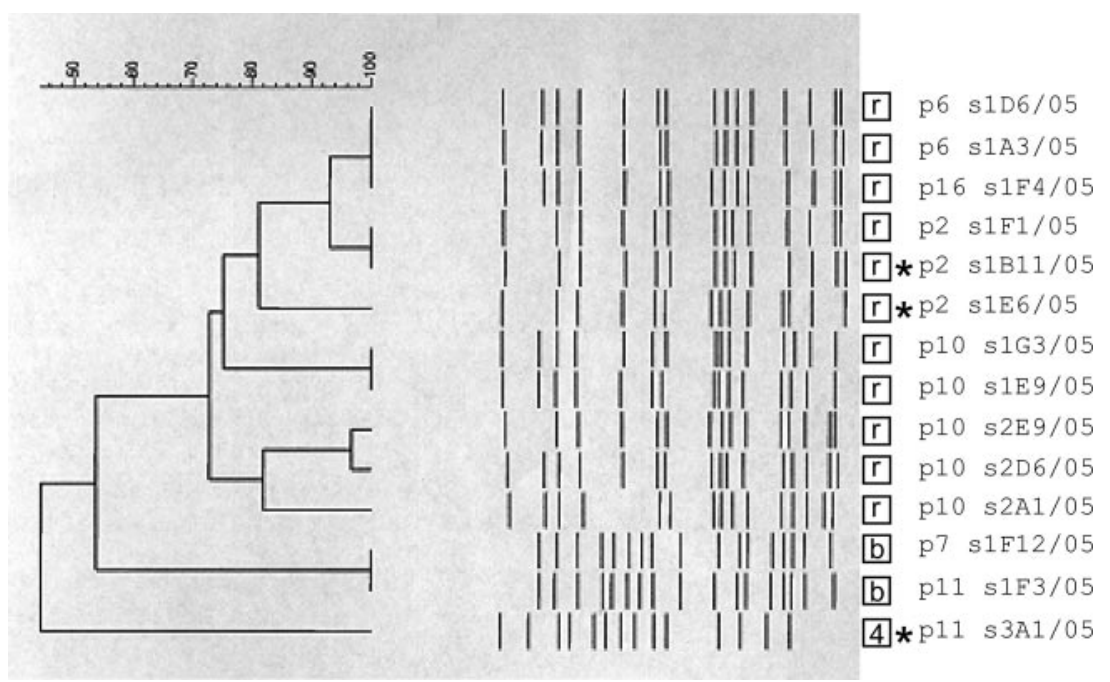

FIG. 4. PFGE analysis of selected $P$. aeruginosa isolates. Labeled boxes indicate specific genotype classes based on SNP typing. Asterisks indicate mucoid isolates.

(presence or absence of these 6 regions) (Fig. 5A). Likewise, the $14 \mathrm{~b}$ clones isolated from different patients were divided into three groups based on the presence or absence of variable regions (Fig. 5B).

This shows that there are genetic variations among clonal isolates from both individual patients and different patients. This diversity probably indicates that adaptations to specific niches occur within the complex CF patient lung environment or that adaptation of $P$. aeruginosa to life in the CF lung is achieved by multiple evolutionary paths. The adaptive significance of the observed diversity will require further characterizations.

The two dominant clones are transmissible. Sequential isolates collected from six of the seven chronically infected patients for periods of 12 to 28 years (Fig. 6) were genotyped in order to investigate the $P$. aeruginosa population dynamics within the patient group. Interestingly, among the 77 archival strains tested, only 10 additional genotypes, not seen in 2005, were found and only 2 of these genotypes were found more than once (genotypes 5 and 6 in patient 2). Again, this overall low level of genotype diversity among the study patients was due to a substantial overrepresentation of the $r$ and $b$ genotypes colonizing the patients. In fact, all six patients have or have had at some point the $\mathrm{b}$ clone, while the $\mathrm{r}$ clone was present in four patients (Fig. 6).

Our longitudinal study also showed that, with the exception of the 14 years of clonal colonization by the $b$ strain in patient 7 , chronic infection appears to be dynamic, resulting in periodic replacement of genotypes with other genotypes (Fig. 6). For example, in patient 2 several strain replacement episodes occurred between 1978 and 2005, resulting in a temporal pattern of establishment and elimination of the 5, 6, and b clones and leaving the $\mathrm{r}$ clone as the dominant clone since 1994. A similar pattern was observed in patients 6 and 10, in which the initial $\mathrm{b}$ clone was replaced by the $\mathrm{r}$ clone. In patients 11 and 16 we observed the establishment of coinfections. In patient 11, the 4 clone was repeatedly found between 1991 and 2003. From that time and onward the b clone was consistently iso-
A

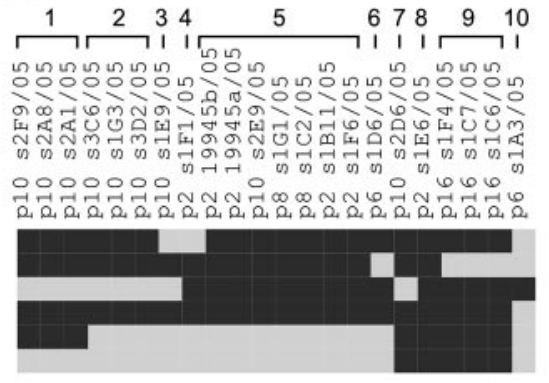

B

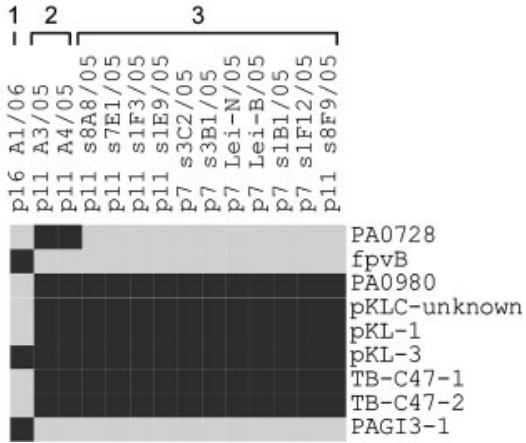

FIG. 5. Genetic variations among clonal isolates. Summary of the genetic variations among isolates from 2005. (A) Twenty-two isolates of the $r$ genotype. (B) Fourteen isolates of the b genotype. The presence or absence of particular genes or regions in different isolates is shown by black and gray boxes, respectively. 


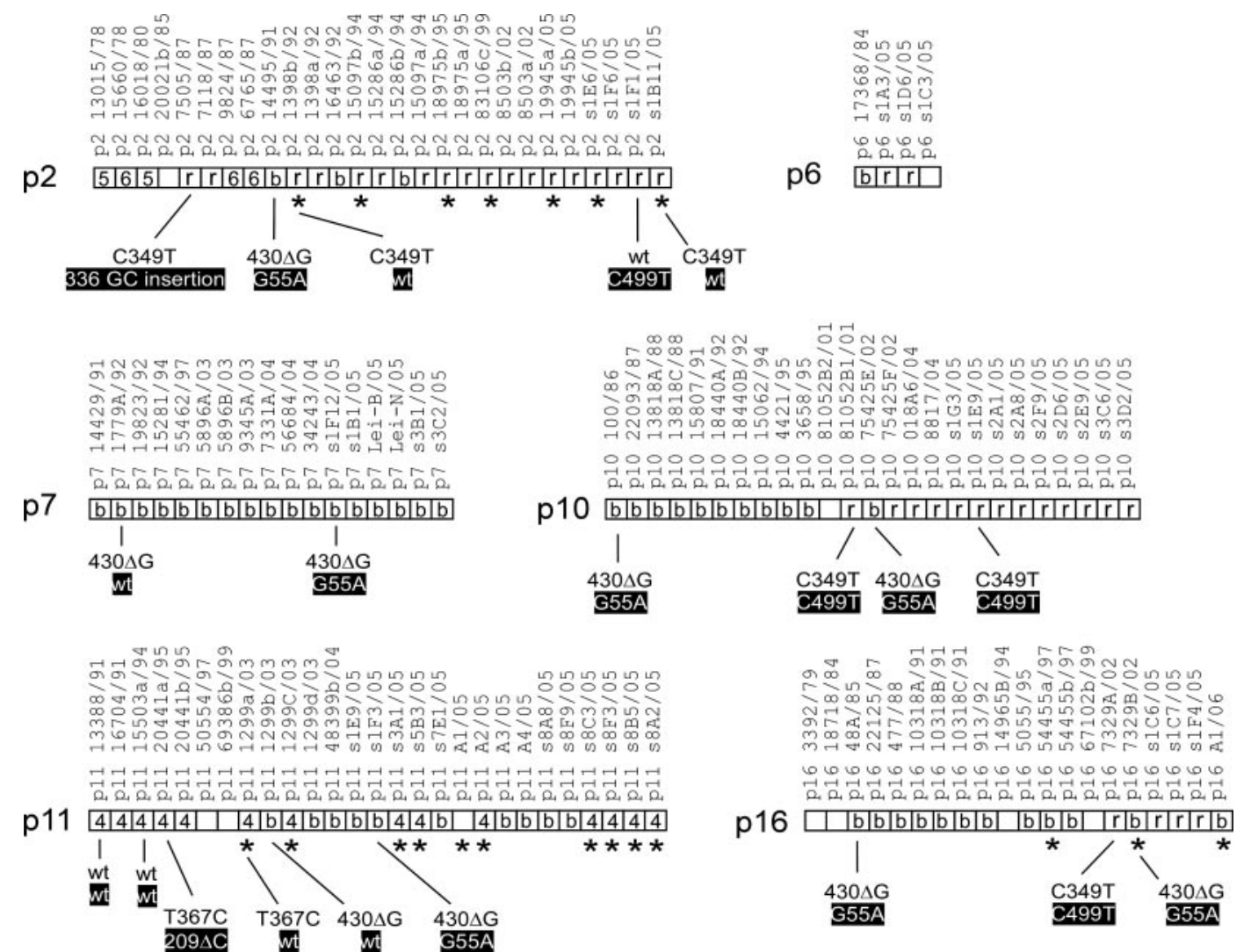

FIG. 6. Genotyping of sequential $P$. aeruginosa isolates from patients with long-term chronic infection. Boxes below the names of the isolates indicate specific genotype classes. White boxes specify unique genotypes found only once in the data set. Labeled boxes specify that a genotype was found more than once. Asterisks indicate mucoid isolates. The results of sequence analysis of $m u c A$ and $a \lg T$ from selected isolates are shown in black and white text, respectively.

lated together with the 4 clone. In patient 16 the $\mathrm{b}$ clone has been isolated since 1985 and has been isolated since 2002 together with the $\mathrm{r}$ clone.

These results clearly show that the two dominant clones are transmissible between patients. We observed a total of 10 infection events among these patients involving either the $\mathrm{r}$ or $\mathrm{b}$ clone among the six patients with long-term infection (Fig. 6). These findings suggest that these particular clones must have strong selective advantages in colonizing CF patient airways.

Transmissibility of the dominant clones is not linked to mucoidy. During the course of CF patient lung infection $P$. aeruginosa usually undergoes a transition from nonmucoid to a mucoid, alginate-overproducing phenotype. We found both nonmucoid and mucoid strains in four of the seven chronically infected patients (Fig. 3 and 6). In patients 2 and 8 nonmucoid and mucoid isolates were of the same genotype whereas in patients 11 and 16 they were of different genotypes (Fig. 6).

It has been shown that overproduction of alginate confers a selective survival advantage for the mucoid $P$. aeruginosa strains relative to nonmucoid strains in mouse lungs $(4,12,41$, 45). Although we observed both nonmucoid and mucoid variants of both the $\mathrm{r}$ and $\mathrm{b}$ dominant genotypes among our seven study patients (Fig. 3 and 6), we nevertheless consistently found only nonmucoid clones of the $b$ and $r$ genotypes in patients 7 and 10, respectively (Fig. 6). Specifically, patient 7 has been colonized by the nonmucoid $\mathrm{b}$ clone for more that 14 years and patient 10 has carried either the $b$ or the $r$ nonmucoid clone for a period of 20 years. Nevertheless, close inspection of the bacteriology records for these two patients showed that the mucoid variant has been found (but not stored) at a low frequency in the histories of the patients. To rule out the possibility of an existing small subpopulation of mucoid variants in these two patients, we inspected multiple sputum samples from patient 7 (9 samples) and patient 10 (15 samples) during 2005 for the presence of mucoid strains. No mucoid variants were found in the samples, and it thus appears that the clear majority of $P$. aeruginosa strains in these two patients are nonmucoid. For the $r$ and $b$ nonmucoid clones in these two patients it appears that adaptive features other than the transition to the mucoid phenotype enable them to persist and establish chronic infections in CF patient lungs.

The majority of nonmucoid isolates are mucoid revertants. Mutations in the mucA gene are known to cause the mucoid phenotype $(4,24)$. To further understand the mucoid/nonmucoid dynamics of the two dominant clones, we determined the DNA sequences of the mucA gene for selected isolates. For the b clone, we sequenced the mucA gene from eight nonmucoid isolates from patients $2,7,10,11$, and 16 and one mucoid isolate from patient 16 (Fig. 6). Interestingly, all nine isolates had the same $430 \Delta \mathrm{G}$ (mucA22) mutation, suggesting that the nonmucoid isolates are in fact revertants that carry a mutation(s) that suppresses the mucA mutations. One candidate 
gene for such a second-site reverting mutation is the $\operatorname{alg} T$ gene (9). We found that eight of the nine isolates also carried a G55A mutation in $\operatorname{alg} T$, resulting in a Glu-to-Lys substitution (Fig. 6). However, as the mucoid isolate $7329 \mathrm{~B} / 02$ from patient 16 also carried this mutation in $\operatorname{alg} T$, it is unlikely to be the only cause of suppression of the mucA mutation.

Sequencing of the mucA gene from five nonmucoid and two mucoid isolates of the $\mathrm{r}$ clone from patients 2,10 , and 16 showed a result similar to that obtained for the b clone (Fig. 6). Six of the seven isolates carried the same C349T mucA mutation, whereas one nonmucoid isolate from 2005 had the wildtype muc $A$ gene. Of the five nonmucoid isolates, four carried a C499T mutation in $\operatorname{alg} T$, resulting in a Pro-to-Ser substitution, and one carried a GC insertion at position 336, which introduced a stop codon at position 340 (Fig. 6). The two mucoid isolates analyzed had no mutations in $\operatorname{alg} T$. Again, these data suggest that nonmucoid isolates are mucA revertants. In contrast to the findings for the $\mathrm{b}$ clone, we cannot exclude mutations in $\operatorname{alg} T$ as the only cause of suppression of mutations in mucA.

Finally, we sequenced the mucA and $\operatorname{alg} T$ genes from three nonmucoid isolates and one mucoid isolate of the 4 genotype from patient 11 (Fig. 6). The two nonmucoid isolates from 1991 and 1994 had wild-type mucA and $\operatorname{alg} T$ sequences (Fig. 6). A nonmucoid isolate from 1995 carried a T367C mucA mutation as well as a $209 \Delta \mathrm{C}$ deletion in the $\operatorname{alg} T$ sequence, which most likely explains the nonmucoid phenotype. Again, this particular isolate appears to be a $m u c A$ revertant. A mucoid isolate from 2003 carried the same T367C mucA mutation and a wild-type $\operatorname{alg} T$ gene.

Taken together, our sequencing results for the mucA gene show that a clear majority of nonmucoid isolates (13 out of 16) contain mutations in mucA and that they consequently are revertants originating from mucoid parent strains. Importantly, none of the 10 infection events involving either the $\mathrm{r}$ or the b clones among the study patients involved mucoid variants (Fig. 6).

The transmissible clones retain their potential to infect $\mathbf{C F}$ patient airways despite reduced virulence. It has previously been shown that transmissible and highly infectious clones such as the Liverpool epidemic strain (LES) exhibit enhanced virulence (37). To further characterize the two transmissible Copenhagen clones, we measured virulence of selected isolates using the C. elegans virulence model (Materials and Methods). As shown in Fig. 7, both an $\mathrm{r}$ clone isolated from patient 2 in 2005 (isolate 19945b/05) and a b clone isolated from patient 7 in 2004 (isolate 7331A/04) were completely nonvirulent in this assay, whereas an unrelated strain from the newly chronically infected patient B3 (isolate s0/04) killed the worms as readily as the virulent control strain. This finding is consistent with previous observations that virulence and virulence factors of $P$. aeruginosa are lost due to mutations during long-term colonization of the CF patient airways $(20,23,40)$. Importantly, we also found a b clone cross-infecting patient 11 in 2003 (isolate $1299 \mathrm{~d} / 03$ ) to be completely nonvirulent in the worm assay (Fig. 7). These results suggest that, although virulence has been reduced for both the $\mathrm{b}$ and $\mathrm{r}$ clones during colonization of Danish CF patients over a period of 20 years, the clones have preserved their capacity to spread among the patients.

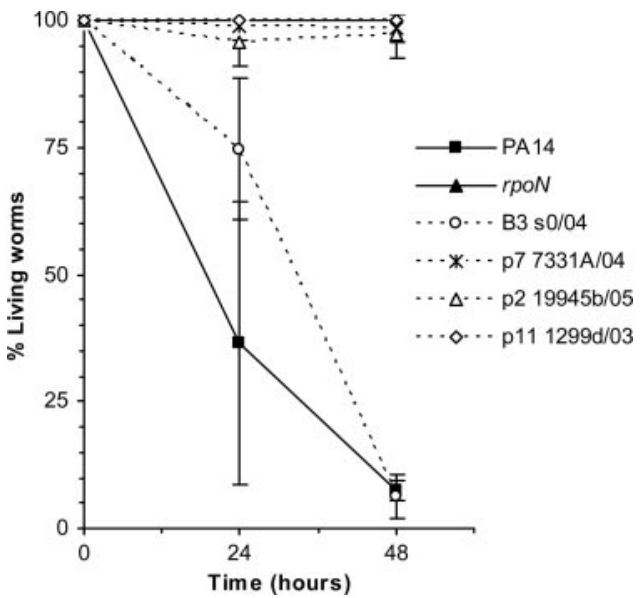

FIG. 7. Survival of $C$. elegans fed on $P$. aeruginosa. C. elegans pha$1(e 2123 t s)$ strain worms were seeded onto bacterial lawns of $P$. aeruginosa isolates B3 s0/04 (open circles), p7 7331A/04, p2 19945b/05, and p11 1299d/03. PA14 and an rpoN mutant were used as virulent and avirulent control strains, respectively.

\section{DISCUSSION}

We have examined the initial $P$. aeruginosa colonization and chronic infection dynamics in a group of CF patients at the Danish CF Center in Copenhagen. We have shown that an overall high level of $P$. aeruginosa genotype diversity characterizes CF patients with intermittent colonization or recently acquired chronic infections and that this reflects the genotype diversity found in other clinical settings and the environment (Fig. 1 and 3). In agreement with previous findings, this suggests that $\mathrm{CF}$ patients acquire unique $P$. aeruginosa strains independently, presumably from different environmental reservoirs $(5,16,35,36)$. In general, these isolates are nonmucoid, virulent, and susceptible to antibiotics. For intermittently colonized patients we furthermore observed that, following initial eradication by antibiotic treatment, recolonization by $P$. aeruginosa occurred, with either a different genotype, suggesting a new environmental source of infection, or with the same genotype, suggesting either undetected colonization (e.g., in the paranasal sinuses) or a persistent environmental source. Longitudinal genotyping of isolates from recently chronically infected patients showed clonal infections and development of the mucoid phenotype, illustrating what is generally thought to be a characteristic pattern of infection of the CF patient airways.

In contrast, genotyping of longitudinal isolates from patients with long-term chronic infection revealed a completely different pattern. We observed a clear overrepresentation of two distinct clones (the $\mathrm{r}$ and $\mathrm{b}$ clones in Fig. 6). All six patients with long-term chronic infection studied in detail have at some point in time been infected by either the $r$ or the $b$ clone or both, and in total for these patients we observed 10 infection events involving these clones (Fig. 6). These findings strongly suggest that the clones are transmissible and have the ability to spread among CF patients. We do not know whether the transmission route is direct (person to person) or indirect (i.e., bacteria from a patient are transiently located at the CF Center and inhaled by another patient). The first observation of the 
transmissible clones was in 1987 for the $\mathrm{r}$ clone (isolate $7505 / 87$ from patient 2) and 1984 for the b clone (isolate 17368/84 from patient 6). Although the two transmissible clones have been present in patients at the Copenhagen CF Center for more than 20 years, they are still transmitted from patient to patient. For example, the $\mathrm{r}$ clones $81052 \mathrm{~B} 1 / 01$ and $7329 \mathrm{a} / 02$ colonized patient 10 and 16 in 2001 and 2002, respectively, and the b clone 1299b/03 colonized patient 11 in 2003 (Fig. 6). So far we have not observed the two dominant clones among other groups of CF patients (intermittently colonized and recently chronically infected), suggesting that the cohort isolation procedures employed at the Danish CF Center in Copenhagen appear to have contained these clones to the older group of patients (long-term chronically infected) and prevented further spreading.

As an alternative explanation to the recent occurrences of either $\mathrm{r}$ or $\mathrm{b}$ clones in patients 10, 11, and 16 (which we interpret as recent transmission events), it might be argued that transmissibility was an original but no longer present trait of the clones, which allowed spreading of the clones among patients some time ago. If so, their sudden and recent appearance in different patients reflects the presence of undetected, indigenous subpopulations of either of the clones, which due to unknown factors (such as changes in treatment strategies, altered airway pathology, etc.) suddenly gained advantages over the present dominant population of $P$. aeruginosa. To disclose the presence of such already-present subpopulations, we specifically searched for small subpopulations of the $r$ clone in patient 7 and for subpopulations of the b clone in patient 10 . Phenotype screening (colony typing and antibiotic resistance profiling) coupled to genotyping by AT biochips of many $P$. aeruginosa isolates from multiple sputum samples taken during 2005 from these two patients failed to provide evidence for the presence of such residual subpopulations (data not shown). We therefore suggest that the two dominant clones have preserved their transmissibility during their presence in the airways of Danish CF patients for more than 20 years and that these clones have strong selective advantages for colonizing $\mathrm{CF}$ patient lungs.

Our data further suggest that the virulence potential of both the $r$ and the $b$ clones plays little if any role in their ability to persist in $\mathrm{CF}$ airways and to spread among patients. In fact, virulence as measured both in the $C$. elegans virulence model (Fig. 7) and by the Bacillus subtilis killing system (32) (data not shown) was severely reduced for both genotypes recently isolated after long-term colonization of specific patients, or after a recent transmission to a different patient. In support of the latter finding, it was recently shown that recolonization of the airways in a CF patient after lung transplantation was by an $r$ clone that was found to be severely reduced in virulence factor production (20). Our findings for the two dominant clones at the Copenhagen CF Center are different from reports concerning other transmissible clones, such as the Liverpool epidemic strain, which display enhanced virulence that could contribute to their spread throughout CF patient populations (37).

We found that the $P$. aeruginosa population structure within the CF patient airway is highly dynamic, even during long-term chronic infection. Although in most patients only one clone or very few clones are present at any given time, we frequently observed replacements of infecting clones (Fig. 6). For exam- ple, in patients 2,6 , and 10 we observed several periods of establishment of infections followed by elimination of specific clones. Equally important, we also observed several infecting clones that failed to establish in the lungs of the patients (Fig. 6 ). The latter clones could be environmental or clinical isolates, which transiently established in the lungs but were competed out by the resident population or eliminated by antibiotics and/or the immune system. Clearly, infection of an already chronically infected $\mathrm{CF}$ patient lung requires special properties. The most noticeable exception from this pattern was patient 7 , who showed a stable infection by a nonmucoid $b$ clone for a period of 14 years.

The $P$. aeruginosa population dynamics in patient 10 is of special interest. In this patient, we observed a complete replacement of the nonmucoid $\mathrm{b}$ clone by the nonmucoid $\mathrm{r}$ clone (Fig. 6). From 1986 to 2002 only the b clone was found to colonize the airways of this patient. From 2002 onward only the $\mathrm{r}$ clone was found. A similar pattern of the $\mathrm{b}$ clone replaced by the $\mathrm{r}$ clone was also observed in patients 2 and 6 (Fig. 6). Changing patient treatment protocols cannot readily explain this eradication of the $\mathrm{b}$ clone, as this particular clone was not eradicated in patients 7, 11, and 16. From an ecological perspective these data suggest that the $r$ and $b$ clones compete in the same specific niches of the CF patient lung and that in the cases of patients 2,6 , and 10 better fitness of the $\mathrm{r}$ clone than of the residing $\mathrm{b}$ clones results in niche exclusion and eradication of the $b$ clone.

Based on these results, we therefore propose that competition among different $P$. aeruginosa genotypes plays a significant role in determining the $P$. aeruginosa population structure within the $\mathrm{CF}$ patient airways. Competition and the presence of two highly adapted and transmissible clones together with the aggressive antibiotic therapy employed at the CF clinic in Copenhagen result in the specific $P$. aeruginosa population structure reported here, in which new incoming clones are unable to establish themselves in the CF patients and alreadypresent clones that cannot be eradicated by conventional therapy in fact can be displaced completely (eradicated) by interclonal competition. Although the specific mechanism of competition is unknown, it is obvious that the nutritional conditions, tolerance to antibiotics and the immune system, and responses to other stress factors could constitute significant competitive factors.

During the course of $\mathrm{CF}$ patient lung infections, $P$. aeruginosa usually undergoes a transition from a nonmucoid to a mucoid phenotype. Significant attention has been given to the emergence of the mucoid phenotype as the presence of mucoid variants is thought to mark the transition to the fatal, chronic stage of the infection (14). The mucoid variants are indeed better protected than nonmucoid bacteria against the inflammatory defense mechanisms of the host and show improved persistence in mouse lungs $(4,6,12,41,45)$. Clinically, the presence of mucoid variants is associated with poor prognosis, deteriorating lung function, and increased tissue damage (33). In agreement with these observations, our longitudinal studies of $P$. aeruginosa isolates from different patients showed the development of the mucoid phenotype during the course of infection. For example, clone 3 in patient B3, clone 4 in patient 11 , the $\mathrm{r}$ clone in patient 2 , and the $\mathrm{b}$ clone in patient 16 all 
developed the mucoid phenotype starting from an initial nonmucoid population.

Nevertheless, in patients 7 and 10 we observed long-term colonization mainly by nonmucoid variants. For the $r$ and $b$ nonmucoid clones in these two patients, mucoidy must be replaced by other adaptive features that provide the bacteria with selective advantages for living in the two specific $\mathrm{CF}$ patient lungs. From an epidemiological point of view, the nonmucoid variants of the $r$ and $b$ genotypes are also of special interest, since all 10 infection events by the $\mathrm{r}$ or $\mathrm{b}$ clone evident in our data set were by nonmucoid variants. Our data thus emphasize that persistence and transmissibility mechanisms of nonmucoid strains associated with the chronic infection process should be further investigated.

From an ecological and evolutionary perspective loss of the transcriptional regulator $m u c A$ by mutation and the resulting conversion to the mucoid phenotype are an example of adaptive evolution that results in ecological specialization by providing the bacteria with adaptive advantages for establishment and survival specifically in the unique CF patient lung niche. Indeed, mucoid variants are rarely if ever isolated as free-living bacteria. In that sense mucoidy can be considered an important virulence factor in case of CF infections. Development of the mucoid phenotype thus resembles the concept of within-host, shortsighted evolution of virulence as proposed by Levin and Bull (21). The model is based on three conditions (21), which are all fulfilled in the case of emergence of mucoid variants during infection of the CF patient lung (1). The bacteria responsible for increased morbidity and mortality of a given host are a result of niche expansion by a genetically distinct subpopulation that arises by mutations within the host during the course of infection (2). The subpopulation becomes established because of local advantages over the ancestral population that they have within the host (3). The selective advantage of the virulent subpopulation is shortsighted because it is uniquely local, that is, within the host. Its members have a disadvantage in their capacity for, or likelihood of, infectious transmission to new hosts. In the extreme case, they are evolutionary dead-ends.

The protection against the immune system provided by the alginate encasement of the mucoid variants probably results in niche expansion, maybe causing infections of the alveoli in the aerobic compartments of the lung as suggested by Høiby (13). The alginate-overproducing mutants, however, are characterized by a reduced fitness in other environmental contexts due to the severe cost of producing the polysaccharide in large amounts, and in this respect they could be viewed as evolutionary dead-ends.

One prediction from the described concept of shortsighted evolution is that evolution of an alginate-overproducing phenotype may open up an alternative niche that is not occupied by the parent cells. This would allow the two different cell lines to coexist stably in the CF patient lung, in agreement with the observations that in patients 2, 11, and 16 we consistently found both mucoid and nonmucoid clones in the sputum samples-in patient 2 derived from the same ancestral clone and in patients 11 and 16 derived from different clones. However, in patients 7 and 10, harboring nonmucoid clones, no stable coexistence of different genotypes was observed. These results indicate that the CF patient lung constitutes more than one ecological niche (the aerobic respiratory zone and the conductive zone containing anaerobic sputum, as suggested by Høiby (13) and that competition between cells does take place within a niche but not between cells in separate niches.

Sequencing of the $m u c A$ gene in several isolates of different genotypes showed that most of the nonmucoid isolates were in fact revertants from mucoid parent strains (13 out of 16 nonmucoid isolates carried mutations in $m u c A$ ). For some of the nonmucoid revertants we found mutations in $\operatorname{alg} T$ that could explain the suppression of the mucA mutation (9), but most isolates seem to harbor suppressor mutations in so-far-unidentified genes. Whatever the genetic mechanisms responsible for suppression of the mucA mutation, we suggest that this is an example of further within-host evolution that consequently results in escape from the potential evolutionary dead-end of being mucoid. The nonmucoid revertants (containing mucA mutations as well as suppressor mutations) must have evolved alternative survival strategies that can substitute for mucoidy. We suggest that these adaptive properties, which could override the significance of mucoidy, might include increased antibiotic resistance, improved utilization of the lung environment, and increased tolerance of the immune response and stress in general. By using these alternative strategies for survival they have retained their ability to spread to new hosts.

In conclusion, this epidemiological study of $P$. aeruginosa isolates from a group of $\mathrm{CF}$ patients associated with the Copenhagen CF Clinic in Denmark has documented the dominance of two distinct genotypes infecting the patients and pointed out cross-infection among the patients as a major cause of the occurrence of these particular clones in connection with chronic lung infections. In most patients these two clones have competed with each other and with other strains, resulting in clonal substitutions at certain time points during the course of the chronic infection. Many patients harbor alginate-overproducing mucoid variants (of either genotype) in coexistence with nonmucoid cells of the same or different genotypes. The data support the theory that these mucoid variant cells occupy a specific niche in the CF patient lung which is separate from that harboring nonmucoid $P$. aeruginosa (13). Further investigations are required to directly document this type of niche specialization.

\section{ACKNOWLEDGMENTS}

This work was supported by a cross-disciplinary research grant from the Danish Research Councils to S.M.

We thank Ulla Rydahl Johansen, Helle Nordbjerg, Katja Bloksted, Pia Poss, and Marianna Baadesby from Rigshospitalet and Tove Johansen from BioCentrum-DTU for excellent technical assistance. We further thank Lütz Wiehlmann for helpful advice in relation to the use of AT tube arrays.

\section{REFERENCES}

1. Al-Aloul, M., J. Crawley, C. Winstanley, C. A. Hart, M. J. Ledson, and M. J. Walshaw. 2004. Increased morbidity associated with chronic infection by an epidemic Pseudomonas aeruginosa strain in CF patients. Thorax 59:334-336.

2. Anthony, M., B. Rose, M. B. Pegler, M. Elkins, H. Service, K. Thamotharampillai, J. Watson, M. Robinson, P. Bye, J. Merlino, and C. Harbour. 2002. Genetic analysis of Pseudomonas aeruginosa isolates from the sputa of Australian adult cystic fibrosis patients. J. Clin. Microbiol. 40:2772-2778.

3. Armstrong, D. S., G. M. Nixon, R. Carzino, A. Bigham, J. B. Carlin, R. M. Robins-Browne, and K. Grimwood. 2002. Detection of a widespread clone of Pseudomonas aeruginosa in a pediatric cystic fibrosis clinic. Am. J. Respir. Crit. Care Med. 166:983-987. 
4. Boucher, J. C., H. Yu, M. H. Mudd, and V. Deretic. 1997. Mucoid Pseudomonas aeruginosa in cystic fibrosis: characterization of muc mutations in clinical isolates and analysis of clearance in a mouse model of respiratory infection. Infect. Immun. 65:3838-3846.

5. Burns, J. L., R. L. Gibson, S. McNamara, D. Yim, J. Emerson, M. Rosenfeld, P. Hiatt, K. McCoy, R. Castile, A. L. Smith, and B. W. Ramsey. 2001 Longitudinal assessment of Pseudomonas aeruginosa in young children with cystic fibrosis. J. Infect. Dis. 183:444-452.

6. Cabral, D. A., B. A. Loh, and D. P. Speert. 1987. Mucoid Pseudomonas aeruginosa resists nonopsonic phagocytosis by human neutrophils and macrophages. Pediatr. Res. 22:429-431.

7. Cheng, K., R. L. Smyth, J. R. Govan, C. Doherty, C. Winstanley, N. Denning, D. P. Heaf, H. van Saene, and C. A. Hart. 1996. Spread of beta-lactamresistant Pseudomonas aeruginosa in a cystic fibrosis clinic. Lancet 348:639642 .

8. Denton, M., K. Kerr, L. Mooney, V. Keer, A. Rajgopal, K. Brownlee, P. Arundel, and S. Conway. 2002. Transmission of colistin-resistant Pseudomonas aeruginosa between patients attending a pediatric cystic fibrosis center Pediatr. Pulmonol. 34:257-261.

9. DeVries, C. A., and D. E. Ohman. 1994. Mucoid-to-nonmucoid conversion in alginate-producing Pseudomonas aeruginosa often results from spontaneous mutations in $a l g T$, encoding a putative alternate sigma factor, and shows evidence for autoregulation. J. Bacteriol. 176:6677-6687.

10. Fluge, G., B. Ojeniyi, N. Høiby, A. Digranes, O. Ciofu, E. Hunstad, O. C Haanaes, and O. T. Storrosten. 2001. Typing of Pseudomonas aeruginosa strains in Norwegian cystic fibrosis patients. Clin. Microbiol. Infect. 7:238243.

11. Frederiksen, B., C. Koch, and N. Høiby. 1997. Antibiotic treatment of initial colonization with Pseudomonas aeruginosa postpones chronic infection and prevents deterioration of pulmonary function in cystic fibrosis. Pediatr. Pulmonol. 23:330-335.

12. Hoffmann, N., T. B. Rasmussen, P. O. Jensen, C. Stub, M. Hentzer, S. Molin, O. Ciofu, M. Givskov, H. K. Johansen, and N. Høiby. 2005. Novel mouse model of chronic Pseudomonas aeruginosa lung infection mimicking cystic fibrosis. Infect. Immun. 73:2504-2514.

13. Høiby, N. 2004. New insight into the pathogenesis and epidemiology of Pseudomonas aeruginosa infection in cystic fibrosis. In H. Goossens, W. Peetermans, and M. Struelens (ed.), Proceedings of the 4th Elzenveld Workshop on Infectious Diseases. Bristol-Myers Squibb, Antwerpen, Belgium.

14. Høiby, N. 1977. Pseudomonas aeruginosa infection in cystic fibrosis. Diagnostic and prognostic significance of Pseudomonas aeruginosa precipitins determined by means of crossed immunoelectrophoresis. A survey. Acta Pathol. Microbiol. Scand. Suppl. 1977:1-96.

15. Høiby, N., and B. Frederiksen. 2000. Microbiology of cystic fibrosis, p 83-107. In M. E. Hodson and D. M. Geddes (ed.), Cystic fibrosis, 2nd ed. Arnold, London, United Kingdom.

16. Jensen, E. T., B. Giwercman, B. Ojeniyi, J. M. Bangsborg, A. Hansen, C. Koch, N. E. Fiehn, and N. Høiby. 1997. Epidemiology of Pseudomonas aeruginosa in cystic fibrosis and the possible role of contamination by dental equipment. J. Hosp. Infect. 36:117-122.

17. Johansen, H. K., and N. Høiby. 1992. Seasonal onset of initial colonisation and chronic infection with Pseudomonas aeruginosa in patients with cystic fibrosis in Denmark. Thorax 47:109-111.

18. Johansen, H. K., L. Norregaard, P. C. Gotzsche, T. Pressler, C. Koch, and N. Høiby. 2004. Antibody response to Pseudomonas aeruginosa in cystic fibrosis patients: a marker of therapeutic success? - a 30-year cohort study of survival in Danish CF patients after onset of chronic $P$. aeruginosa lung infection. Pediatr. Pulmonol. 37:427-432.

19. Jones, A. M., J. R. Govan, C. J. Doherty, M. E. Dodd, B. J. Isalska, T. N. Stanbridge, and A. K. Webb. 2001. Spread of a multiresistant strain of Pseudomonas aeruginosa in an adult cystic fibrosis clinic. Lancet 358:557558.

20. Lee, B., J. A. Haagensen, O. Ciofu, J. B. Andersen, N. Høiby, and S. Molin. 2005. Heterogeneity of biofilms formed by nonmucoid Pseudomonas aeruginosa isolates from patients with cystic fibrosis. J. Clin. Microbiol. 43:52475255 .

21. Levin, B. R., and J. J. Bull. 1994. Short-sighted evolution and the virulence of pathogenic microorganisms. Trends Microbiol. 2:76-81.

22. Mahenthiralingam, E., M. E. Campbell, J. Foster, J. S. Lam, and D. P. Speert. 1996. Random amplified polymorphic DNA typing of Pseudomonas aeruginosa isolates recovered from patients with cystic fibrosis. J. Clin. Microbiol. 34:1129-1135.

23. Mahenthiralingam, E., M. E. Campbell, and D. P. Speert. 1994. Nonmotility and phagocytic resistance of Pseudomonas aeruginosa isolates from chronically colonized patients with cystic fibrosis. Infect. Immun. 62:596-605.

24. Martin, D. W., M. J. Schurr, M. H. Mudd, J. R. Govan, B. W. Holloway, and
V. Deretic. 1993. Mechanism of conversion to mucoidy in Pseudomonas aeruginosa infecting cystic fibrosis patients. Proc. Natl. Acad. Sci. USA 90 8377-8381.

25. McCallum, S. J., M. J. Gallagher, J. E. Corkill, C. A. Hart, M. J. Ledson, and M. J. Walshaw. 2002. Spread of an epidemic Pseudomonas aeruginosa strain from a patient with cystic fibrosis (CF) to non-CF relatives. Thorax 57:559560

26. Morales, G., L. Wiehlmann, P. Gudowius, C. van Delden, B. Tummler, J. L. Martinez, and F. Rojo. 2004. Structure of Pseudomonas aeruginosa populations analyzed by single nucleotide polymorphism and pulsed-field gel electrophoresis genotyping. J. Bacteriol. 186:4228-4237.

27. Munck, A., S. Bonacorsi, P. Mariani-Kurkdjian, M. Lebourgeois, M. Gerardin, N. Brahimi, J. Navarro, and E. Bingen. 2001. Genotypic characterization of Pseudomonas aeruginosa strains recovered from patients with cystic fibrosis after initial and subsequent colonization. Pediatr. Pulmonol. 32:288-292.

28. Nixon, G. M., D. S. Armstrong, R. Carzino, J. B. Carlin, A. Olinsky, C. F. Robertson, and K. Grimwood. 2001. Clinical outcome after early Pseudomonas aeruginosa infection in cystic fibrosis. J. Pediatr. 138:699-704.

29. O'Carroll, M. R., M. W. Syrmis, C. E. Wainwright, R. M. Greer, P. Mitchell, C. Coulter, T. P. Sloots, M. D. Nissen, and S. C. Bell. 2004. Clonal strains of Pseudomonas aeruginosa in paediatric and adult cystic fibrosis units. Eur. Respir. J. 24:101-106.

30. Ojeniyi, B., B. Frederiksen, and N. Høiby. 2000. Pseudomonas aeruginosa cross-infection among patients with cystic fibrosis during a winter camp. Pediatr. Pulmonol. 29:177-181.

31. Ojeniyi, B., U. S. Petersen, and N. Høiby. 1993. Comparison of genome fingerprinting with conventional typing methods used on Pseudomonas aeruginosa isolates from cystic fibrosis patients. APMIS 101:168-175.

32. Park, S. Y., Y. J. Heo, Y. S. Choi, E. Deziel, and Y. H. Cho. 2005. Conserved virulence factors of Pseudomonas aeruginosa are required for killing Bacillus subtilis. J. Microbiol. 43:443-450.

33. Pedersen, S. S., N. Høiby, F. Espersen, and C. Koch. 1992. Role of alginate in infection with mucoid Pseudomonas aeruginosa in cystic fibrosis. Thorax 47:6-13

34. Pedersen, S. S., C. Koch, N. Høiby, and K. Rosendal. 1986. An epidemic spread of multiresistant Pseudomonas aeruginosa in a cystic fibrosis centre. J. Antimicrob. Chemother. 17:505-516.

35. Romling, U., B. Fiedler, J. Bosshammer, D. Grothues, J. Greipel, H. von der Hardt, and B. Tummler. 1994. Epidemiology of chronic Pseudomonas aeruginosa infections in cystic fibrosis. J. Infect. Dis. 170:1616-1621.

36. Romling, U., J. Wingender, H. Muller, and B. Tummler. 1994. A major Pseudomonas aeruginosa clone common to patients and aquatic habitats. Appl. Environ. Microbiol. 60:1734-1738.

37. Salunkhe, P., C. H. Smart, J. A. Morgan, S. Panagea, M. J. Walshaw, C. A. Hart, R. Geffers, B. Tummler, and C. Winstanley. 2005. A cystic fibrosis epidemic strain of Pseudomonas aeruginosa displays enhanced virulence and antimicrobial resistance. J. Bacteriol. 187:4908-4920.

38. Schnabel, H., and R. Schnabel. 1990. An organ-specific differentiation gene, pha-1, from Caenorhabditis elegans. Science 250:686-688.

39. Scott, F. W., and T. L. Pitt. 2004. Identification and characterization of transmissible Pseudomonas aeruginosa strains in cystic fibrosis patients in England and Wales. J. Med. Microbiol. 53:609-615.

40. Smith, E. E., D. G. Buckley, Z. Wu, C. Saenphimmachak, L. R. Hoffman, D. A. D'Argenio, S. I. Miller, B. W. Ramsey, D. P. Speert, S. M. Moskowitz J. L. Burns, R. Kaul, and M. V. Olson. 2006. Genetic adaptation by Pseudomonas aeruginosa to the airways of cystic fibrosis patients. Proc. Natl. Acad. Sci. USA 103:8487-8492.

41. Song, Z., H. Wu, O. Ciofu, K. F. Kong, N. Høiby, J. Rygaard, A. Kharazmi, and K. Mathee. 2003. Pseudomonas aeruginosa alginate is refractory to Th1 immune response and impedes host immune clearance in a mouse model of acute lung infection. J. Med. Microbiol. 52:731-740.

42. Taccetti, G., S. Campana, F. Festini, M. Mascherini, and G. Doring. 2005 Early eradication therapy against Pseudomonas aeruginosa in cystic fibrosis patients. Eur. Respir. J. 26:458-461.

43. Tan, M. W., S. Mahajan-Miklos, and F. M. Ausubel. 1999. Killing of Caenorhabditis elegans by Pseudomonas aeruginosa used to model mammalian bacterial pathogenesis. Proc. Natl. Acad. Sci. USA 96:715-720.

44. Thomsen, L. E., S. S. Slutz, M. W. Tan, and H. Ingmer. 2006. Caenorhabditis elegans is a model host for Listeria monocytogenes. Appl. Environ Microbiol. 72:1700-1701.

45. Yu, H., M. Hanes, C. E. Chrisp, J. C. Boucher, and V. Deretic. 1998 Microbial pathogenesis in cystic fibrosis: pulmonary clearance of mucoid Pseudomonas aeruginosa and inflammation in a mouse model of repeated respiratory challenge. Infect. Immun. 66:280-288. 